Article

\title{
Predictive Set Point Modulation Charging of Autonomous Rail Transit Vehicles
}

\author{
Heng Li ${ }^{1}$, Yu Zhang ${ }^{1}$, Hongtao Liao ${ }^{2, *}$ and Jun Peng ${ }^{1}$ (D) \\ 1 School of Computer Science and Engineering, Central South University, Changsha 410083, China; \\ liheng@csu.edu.cn (H.L.); zhang.yu@csu.edu.cn (Y.Z.); pengj@csu.edu.cn (J.P.) \\ 2 School of Automation, Central South University, Changsha 410083, China \\ * Correspondence: lht2015@csu.edu.cn
}

Received: 11 August 2020; Accepted: 18 September 2020; Published: 23 September 2020

\begin{abstract}
Autonomous rail transit (ART) vehicle is a new type of urban rail transportation, which has good development prospects. It is powered by onboard supercapacitors, which are charged at midway stations. It requires short charging time and fast charging speed. Usually, multiple chargers are used in parallel for charging. However, this will cause an overshoot phenomenon during charging, and the overshoot of multiple chargers will be superimposed on the supercapacitor, affecting the stability and life of both supercapacitors and chargers. In this paper, we propose a predictive set point modulation charging method, which can reduce the system's overshoot and increase the reliability of the system. First, the state-space averaging method is used to establish the electronic physical model of the multicharger system. Secondly, a predictive set point modulation charging control method is designed, and the closed-loop model of the proposed charging system is developed using the buck diagram. The effectiveness of the proposed method is verified through extensive simulation and experiments. The experimental results show that compared with the classical design method, the proposed method can effectively suppress the current overshoot.
\end{abstract}

Keywords: ART; supercapacitor; multiple chargers; overshoot; set point modulation

\section{Introduction}

The global energy market is currently in a transition period. The rapid growth and accelerated prosperity of energy demand come more from developing countries, such as China and India [1,2]. China plans to have 5 million new energy vehicles in 2020, and new energy vehicles have great development potential. According to media reports, in 20 years, China will stop selling traditional fuel vehicles and replace them with new energy vehicles powered by pure electricity. It is expected that the world will stop selling fuel vehicles around 2050, and a new chapter in the new energy vehicle industry will be opened [3,4]. Therefore, the battery of new energy vehicles has become an important factor hindering their development.

The world's first autonomous rail rapid transit (ART) vehicle was unveiled in Zhuzhou Locomotive Co. Ltd. on 2 June 2017. Since May 2018, ART has started test operations in Zhuzhou, China. ART vehicles are used between buses and trams. They use rubber tires and a white line painted with a two-dot chain line along the road instead of traditional railroad tracks. Moreover, the ART vehicle is totally powered by onboard energy storage systems without any overhead catenaries.

Compared with existing bus rapid transit (BRT) and the light rail transit (LRT), the ART provides some distinctive advantages in ride quality, land development potential, and cost. Table 1 [5] shows the comparison results of the three public transportation systems. ART has many advantages, it can complement the existing public transportation system and is expected to play an important role in the future public transportation. 
Table 1. Comparisons of bus rapid transit (BRT), light rail transit (LRT), and Autonomous rail transit (ART) systems.

\begin{tabular}{cccc}
\hline Metric & BRT & LRT & ART \\
\hline Capacity and velocity & + & ++ & ++ \\
Ride quality & - & ++ & ++ \\
Land development prospect & - & ++ & ++ \\
Expense & + & - & + \\
Disruption during construction period & + & - & + \\
Construction time & + & - & + \\
Overall rating & + & ++ & +++ \\
\hline
\end{tabular}

Battery and supercapacitor are two main energy storage systems for ART vehicles. The battery needs a long charging time and it has a large storage capacity. The supercapacitor can complete the charge and discharge process in a short time, but its storage capacity is poor. The public transportation system has the characteristics of frequent parking and short distance from station to station, and ART based on supercapacitor is more popular in urban areas.

Charging systems are crucial for the operation of ART, since they are the only power source of onboard supercapacitors. The ART vehicle can be charged at stations, i.e., midway charging. When passengers get on/off the vehicle, the vehicle needs to be charged quickly. During the charging process, the charger has a large power, and a single charger cannot meet the requirements.

In order to improve the charging efficiency, we connect multiple chargers in parallel to charge the supercapacitors. We use decentralized control for chargers. The method of decentralized control has the advantages of working independently and not affecting the others [6]. It has high reliability, reasonable system structure, and simple programming. It is easy to modify and change due to the reduction of the amount of information. It will improve the efficiency of the chargers.

Current overshooting is undesired in the charging system design. During the start-up phase, when the supplied current $0 \mathrm{~A}$ is changed to the required current, the set-point jump causes the current overshoot. From the control's perspective, in the second-order system, we generally choose a damping ratio of $0.4 \sim 0.8$. When the damping ratio of the system is too small, this will cause current overshoot. When a large damping ratio is selected, although the response overshoot can be avoided, it will extend the settling time and has no effect on practical applications. We use multiple chargers in parallel, and the overshoot phenomenon will be superimposed on the super capacitor, which will greatly reduce the life and stability of the chargers and super capacitors $[7,8]$.

The method of set point modulation is a popular technique to get a quick (that is, shorter stay times) and smooth (that is, shorter overtaking times) response [9-11]. It has been widely used in power systems, for example micro-grids and active distribution system (ADS) [12]. To improve the performance of the prediction algorithm, predictive set point modulation uses patterns to predict future response values, for which it predicts and stores samples at the sampling rate [13].

The main contributions of this article consist of the following three aspects.

- We propose a predictive set point modulation charging method to reduce the system overshoot and increase the life of chargers and supercapacitors.

- The mathematical model of the multiple charger is established through the transfer function, and the controller parameters are explicitly calculated by the classical control theory.

- Extensive simulations and experiments are provided to verify our design. It can reduce the overshoot phenomenon during the charging process.

The specific structure of this article is as follows. Section 2 describes how to build a mathematical model of the multifunctional charger system. In Section 3, we propose a predictive set point modulation charging method. Section 4 provides specific case studies. We summarized this paper in Section 5. 


\section{System Modeling}

In this section, we model the charging system mathematically [14]. Specifically, the state-space averaging method is used to model the physical layer and the transfer function matrix model is derived. We choose the buck converter as the main topology, because the buck converter has obvious advantages such as simple structure and good dynamic performance. The detailed layout of the multiple charger is shown in Figure 1. The supercapacitor is modeled as an resistor-capacitor (RC) circuit, where $R$ is the internal resistance of the super capacitor, and $C$ represents the capacitance. In the equivalent RC model of supercapacitors, the equivalent capacitance represents the energy storage effect, and the equivalent resistance represents the power loss effect. In practical applications, the power grid voltage is alternative current (AC) $380 \mathrm{~V}$, and the frequency is $50 \mathrm{~Hz}$. An isolated transformer $(400 \mathrm{~V} / 200 \mathrm{~V})$ is typically employed for the isolation, voltage matching, and filtering. The diode-based pulse-width-modulation (PWM) rectifier is used to convert the AC input to direct current (DC) signals. Detailed physical setting can be referred to [15].

We connect $\mathrm{n}$ chargers in parallel and use their current to charge the supercapacitor. The charger is chosen as the startup controller because it has an excellent function of providing high output streams [16]. The supercapacitor is modeled as an RC circuit. The resistance $R$ is the internal resistance of the supercapacitor, which represents the loss of power. The capacitor $C$ represents the storage capacity of circuit.

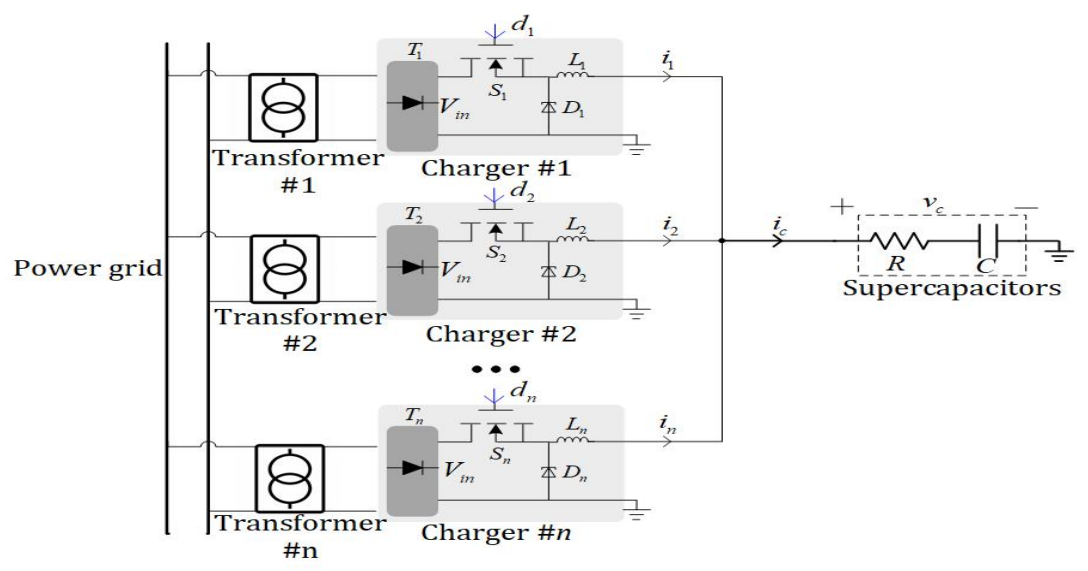

Figure 1. The layout of the multicharger system.

For each $k$ charger, the rectifier $T_{k}$ corrects the AC input of the grid to a DC flow. The DC flow is controlled by pulse width modulation (PWM). The averaging approach is a common approach for the modeling of converters, where the switching frequency is assumed to be much higher than the converter dynamics. Based on the averaging approach, the physical model is obtained as [17]

$$
\left\{\begin{array}{l}
L_{k} \frac{d i_{k}}{d t}=V_{i n} d_{k}-v_{c}, k=1,2, \cdots, n \\
C \frac{d v_{c}}{d t}=R C \frac{d i_{c}}{d t}+i_{c}
\end{array}\right.
$$

where $L_{k}, i_{k}, d_{k}$ are the inductance, output current, and duty cycle of charger $k, V_{i n}$ represents the rectified input voltage, $v_{c}$ represents the voltage of the ultracapacitor. $i_{c}=i_{1}+i_{2} \cdots+i_{n}$ is the sum of multiple charger currents, which is used to charge the ultracapacitor.

The state-space model in Equation (1) is a time-domain model. The charging system is represented by $n+1$ differential equations. We use the Laplace transform to complete the conversion from the time domain model to the frequency domain model, and then create the cost system function module to transition from the working cycle of $d_{k}$ to the traditional $i_{k}$. The transfer function module greatly simplifies the design process [18]. 
Proposition 1. The transfer function matrix $G_{i d}(s)$ is derived.

$$
G_{i d}(s)=V_{i n} \Psi^{-1}
$$

where $\Psi=$

$$
\left[\begin{array}{cccc}
s L_{1}+R_{e q}+\frac{1}{s C_{e q}} & R_{e q}+\frac{1}{s C_{e q}} & \cdots & R_{e q}+\frac{1}{s c e q} \\
R_{e q}+\frac{1}{s C e q} & s L_{2}+R_{e q}+\frac{1}{s C e q} & \cdots & R_{e q}+\frac{1}{s C_{e q}} \\
\vdots & \ddots & \ddots & \vdots \\
R_{e q}+\frac{1}{s C_{e q}} & R_{e q}+\frac{1}{s C_{e q}} & \cdots & s L_{n}+R_{e q}+\frac{1}{s C_{e q}}
\end{array}\right]
$$

where $R_{e q}$ and $C_{e q}$ represent the equivalent resistance and equivalent capacitance of the supercapacitor.

Proof. Using Laplace transform to Equation (1), we can get

$$
\left\{\begin{array}{l}
s L_{k} I_{k}(s)=V_{i n} D_{k}(s)-V_{c}(s), k=1,2, \cdots, n \\
s C_{e q} V_{c}(s)=s R_{e q} C_{e q} I_{c}(s)+I_{c}(s),
\end{array}\right.
$$

where $V_{c}(s), I_{\mathcal{C}}(s)$ are the Laplace transforms of terminal voltage $v_{c}$ and charging current $i_{c}$, respectively. $I_{k}(s)$ is the Laplace transforms of output current $i_{k}$ and $D_{k}(s)$ is the duty cycle $d_{k}$ of the charging module $k$, respectively [19].

The ground frequency model of the load model and the supercapacitor battery are connected together by the terminal voltage $V_{c}(s)$. Then in Equation (3), by deleting the variable $V_{c}(s)$ as Equation (4):

$$
s L_{k} I_{k}(s)+R_{e q} I_{\mathcal{C}}(s)+\frac{1}{s C_{e q}} I_{\mathcal{c}}(s)=V_{i n} D_{k}(s), k=1,2, \cdots, n
$$

It is difficult to directly obtain the transfer function of $D_{k}(s)$ to $I_{k}(s)$ from Equation (4) because the traditional output $I_{k}(s)$ is connected to the others through a conventional load $I_{\mathcal{c}}(s)$. Therefore, we rewrote the frequency model from Equation (4) in a centralized form.

Define the matrix form of system output current $I$, reference current $I^{\prime}$, and input duty cycle $D$ respectively.

$$
\begin{aligned}
& I=\left[\begin{array}{llll}
i_{1} & i_{2} & \cdots & i_{n}
\end{array}\right]^{\mathrm{T}} \\
& I^{\prime}=\left[\begin{array}{llll}
i_{k 1}^{\prime} & i_{k 2}^{\prime} & \cdots & i_{k n}^{\prime}
\end{array}\right]^{T}, \\
& D=\left[\begin{array}{llll}
d_{1} & d_{2} & \cdots & d_{n}
\end{array}\right]^{\mathrm{T}} .
\end{aligned}
$$

Then, Equation (4) is derived into matrix form

$$
\Psi I(s)=V_{i n} D(s),
$$

where $I(s), D(s)$ are the Laplace transforms of $I$ and $D$. The transfer function matrix $G_{i d}(s)$ can be derived as

$$
G_{i d}(s)=V_{i n} \Psi^{-1}
$$

This completes the proof.

\section{Predictive Set Point Modulation Charging}

In this section, we propose a predictive set point modulation charging method and build a closed-loop transfer function model. 


\subsection{Predictive Set Point Modulation}

The predictive set point modulation method can change the charger reference current in real time. The compensator is designed to reduce the transition between the output current $i_{k}$ and the reference $i_{0}$. It can reduce the overshoot of each charger. The four steps of the method are briefly described below. Some specific design steps and parameter selection can be found in [9-11].

The first step is to calculate the deviation between the reference current $i_{0}$ and the actual output current $i_{k}$. So we first calculate the deviation signal $e_{k}(t)$ :

$$
e_{k}(t)=i_{0}-i_{k}(t) \quad k=1,2, \ldots, n
$$

The current deviation signal $e_{k}(t)$ is used to predict the future deviation signal $p_{k}(t)$. The compensator is designed to calculate the predicted deviation signal and suppress the overshoot current.

$$
p_{k}(s)=\frac{s T+1}{\alpha s T+1} e_{k}(s), \quad \alpha<1
$$

where $e_{k}(s)$ and $p_{k}(s)$ are the Laplace transforms of $e_{k}(t)$ and $p_{k}(t)$. The compensator can correct the overshoot within the range of $\left[\frac{1}{T}, \frac{1}{\alpha T}\right]$, where the maximum overshoot occurs at the intermediate frequency $\omega_{m}$. The $\omega_{m}$ can be obtained in the following way:

$$
\omega_{m}=\frac{1}{T} \sqrt{\frac{1}{\alpha}} .
$$

According to the predicted deviation signal in Equation (8), the adjustment signal $m_{k}(t)$ is defined as

$$
m_{k}(t)=m \times p_{k}(t),
$$

it will be used to regulate the reference current. A reasonable $m$ can reduce system overshoot. The specific parameter design is based on existing papers, among which $\alpha=0.2$ is designed based on experience, the sampling period $T=0.02$ can ensure the accuracy, and $m=0.2$ can improve the response speed of the system $[10,11]$.

The reference current $i_{0}$ is modulated to the output current $i_{k}$ in the following way

$$
i_{k}^{\prime}(t)=\left\{\begin{array}{l}
i_{0}, \quad e_{\min } \leqslant p_{k}(t) \leqslant e_{\max } \\
i_{0}+m_{k}(t), \quad \text { otherwise }
\end{array}\right.
$$

When the predicted deviation signal exceeds the allowable range, the reference value is adjusted. The range is defined by $e_{\min }$ and $e_{\max }$. With the predictive set point modulation method in Equations (7)-(11), the output current can be adjusted in real time.

\subsection{Charging Control}

In a feedback control system [20], the tracking error is chosen as a reference signal subtracting the feedback signal. Then, a current tracker is designed as

$$
\delta_{k}=i_{k}^{\prime}-i_{k}
$$

where $\delta_{k}$ is the tracking error, $i_{k}^{\prime}$ is the modulated reference current of charger $k$, and $i_{k}$ is the output current of charger $k$. 
The purpose of the charging control is to guarantee the output current follows the reference current. To do that, a compensator generates the corresponding duty cycle according to the tracking error (12). A proportional integral (PI) compensator $C(s)$ is designed as

$$
C(s)=k_{p}+\frac{k_{i}}{s}
$$

where $k_{i}$ is the integral coefficient and $k_{p}$ is the proportional coefficient [21]. In the following, we derive the closed-loop model of the charging control system.

\subsection{Closed-Loop Modeling}

With the interaction of the model between the physical layer and the control layer, we use the matrix vector representation to derive the closed mesh model. The tracking error $\Delta$ is defined:

$$
\Delta=\left[\begin{array}{llll}
\delta_{1} & \delta_{2} & \cdots & \delta_{n}
\end{array}\right]^{T}
$$

The $\Delta$ can be expressed as

$$
\Delta=\left(I^{\prime}-I\right)
$$

Figure 2 describes the closed-loop model of the charger system. The PSPM (predictive set point modulation) represents the algorithm proposed in Section 3.1. $I_{0}$ and $I$ respectively represent the input current and the output current. The duty cycle $D$ is generated through the compensator $C(s)$. The current output vector $I$ is generated by the transfer function matrix [22].

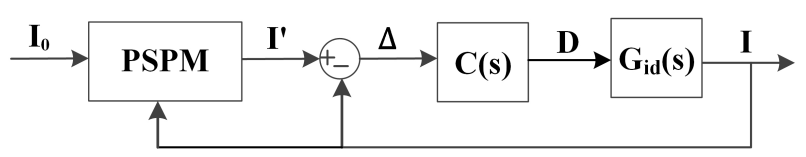

Figure 2. The closed-loop model of the multicharger system.

\section{Case Study}

In this section, the specific controller parameter selection is first derived, and the verification of the charging method is completed through a combination of simulation and experiment.

\subsection{Parameter Setting}

Physical Parameters: The circuit with three chargers in parallel. The specific parameters are designed as follows: the capacitance of ultracapacitors $C=100 \mathrm{~F}$, the ESR of ultracapacitors $R=5 \mathrm{~m} \Omega$, input voltage $V_{\text {in }}=24 \mathrm{~V}$, the nominal inductance of three inductors $L_{1}=L_{2}=L_{3}=1 \mathrm{mH}$ and reference current $i_{0}=1 \mathrm{~A}$.

\subsection{Compensator Design}

First design the open-loop transfer function matrix

$$
G_{o p}(s)=G_{i d}(s) C(s),
$$

then derive the closed-loop transfer function matrix

$$
G_{c l}(s)=\left[\mathbb{I}+G_{o p}(s)\right]^{-1} G_{o p}(s) .
$$


With the circuit parameters, we use MATLAB (Mathworks, Natick, MA, USA) symbolic toolbox [23] to complete the derivation of the transfer function.

$$
\left[\begin{array}{c}
I_{1}(s) \\
I_{2}(s) \\
I_{3}(s)
\end{array}\right]=\left[\begin{array}{lll}
G_{11}(s) & G_{12}(s) & G_{13}(s) \\
G_{21}(s) & G_{22}(s) & G_{23}(s) \\
G_{31}(s) & G_{32}(s) & G_{33}(s)
\end{array}\right]\left[\begin{array}{c}
I_{0}(s) \\
I_{0}(s) \\
I_{0}(s)
\end{array}\right]
$$

where $G_{k m}(s) \forall k, m \in\{1,2,3\}$ is the $(k, m)$ component of the transfer function matrix $G_{c l}(s)$ in (17). The closed-loop transfer function $G_{k 0}(s)=G_{k 1}(s)+G_{k 2}(s)+G_{k 3}(s)$ is derived as

$$
G_{k 0}(s)=\frac{\left(24,000 k_{p}\right) s+24,000 k_{i}}{s^{2}+\left(24,000 k_{p}+15\right) s+24,000 k_{i}+30}
$$

The model $G_{k 0}(s)$ is a second-order transfer function. In order to analyze the performance of the controller, the frequency $\omega_{n}$ and the damping ratio $\zeta$ are calculated as

$$
\omega_{n}=\sqrt{24,000 k_{i}+30}, \zeta=\frac{24,000 k_{p}+15}{2 \sqrt{24,000 k_{i}+30}} .
$$

The time required for the system to stabilize within $4 \%$ of the reference value is called the settling time. The settling time of the system is calculated as [24]

$$
t_{s}=\frac{4.4}{\zeta \omega_{n}}
$$

We set the settling time $t_{s}=0.1$. This is an overdamping system, and we choose $\zeta=0.7$. Substituting the settling time and damping ratio into Equations (19) and (21), we have

$$
k_{p}=0.003, k_{i}=0.163
$$

This completes the design of the compensator.

\subsection{Simulation Results}

We will explore the capability of the proposed charging method. In order to make the experiment more accurate, we used MATLAB (Mathworks, Natick, USA) to perform model. We recorded the corresponding waveform diagrams and combined them with the experimental part below. We designed two sets of experiments for comparison. The first one is a classical second-order PI controller, and the other is the proposed method. We observe the waveform of the output signal and analyze it from the three perspectives of speed, stability, and accuracy.

Figure 3 shows the simulation results, which explores the effect of set point modulation on the charging process. Figure 3a shows the simulation results of the classical charging method, where the overshoot is about $15 \%$, the settling time is about $0.08 \mathrm{~s}$. Figure $3 \mathrm{~b}$ shows the experiment results of the proposed method, where the current overshoot is about $2 \%$, the settling time is about $0.06 \mathrm{~s}$. The proposed charging method is faster than the classical charging method, and the overshooting can be restrained with the proposed charging method.

Figure 4 shows the simulation results of the two methods in total charging current. Figure $4 \mathrm{a}$ shows the simulation results of the classical charging method, where the overshoot is about $15 \%$, and the settling time is about $0.08 \mathrm{~s}$. Figure $4 \mathrm{~b}$ shows the simulation results of the proposed charging method, where the overshoot is about $2 \%$, and the settling time is about $0.06 \mathrm{~s}$. Simulation results verify that the proposed charging method can restrain the current overshoot while reducing the settling time. 


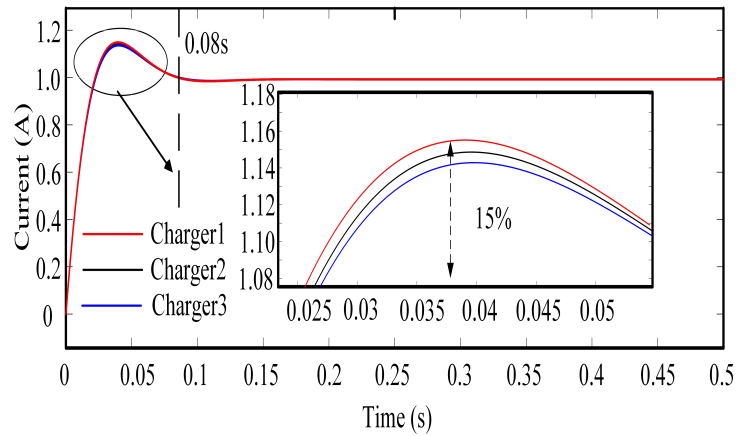

(a)

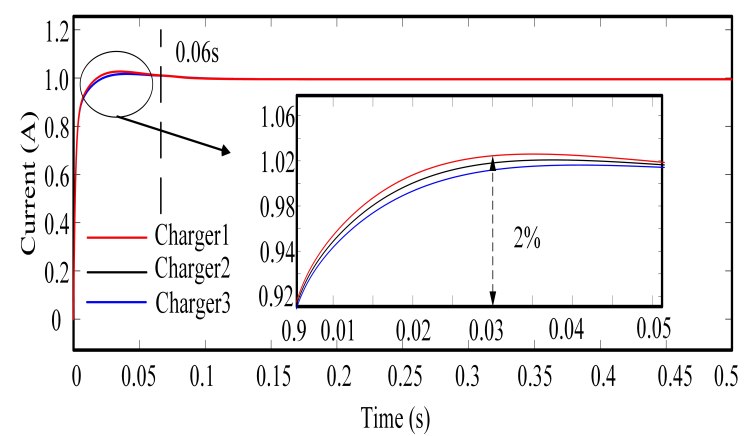

(b)

Figure 3. Simulation results of (a) the classical charging method and (b) the proposed charging method.

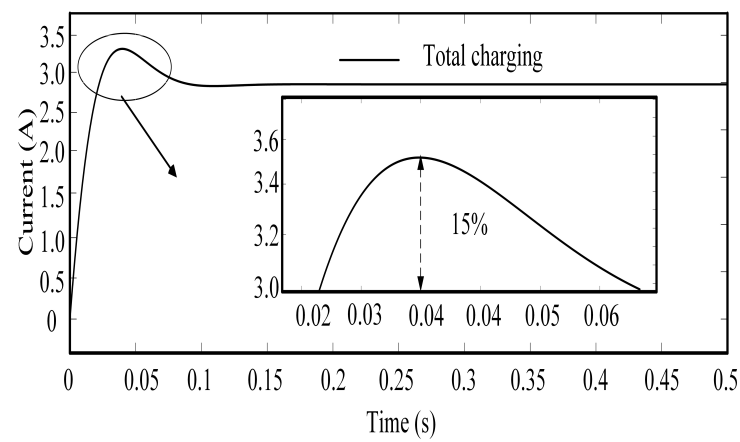

(a)

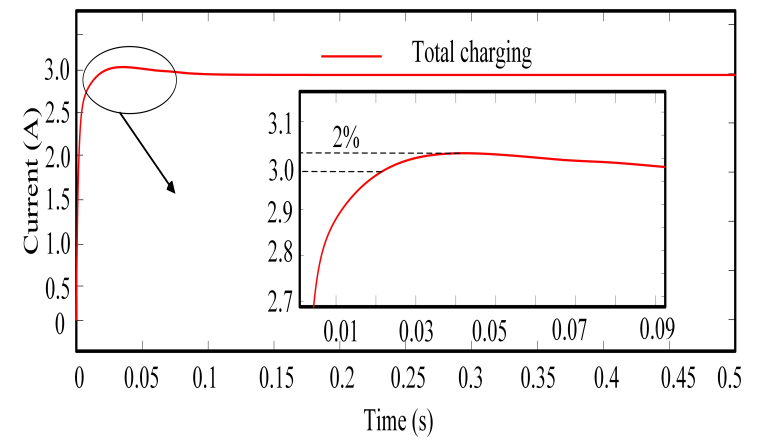

(b)

Figure 4. Simulation results of (a) the classical charging method and (b) the proposed charging method in total charging current.

\subsection{Hardware Setup}

The hardware setup for this experiment is shown in Figure 5. The main components of the test board are as follows

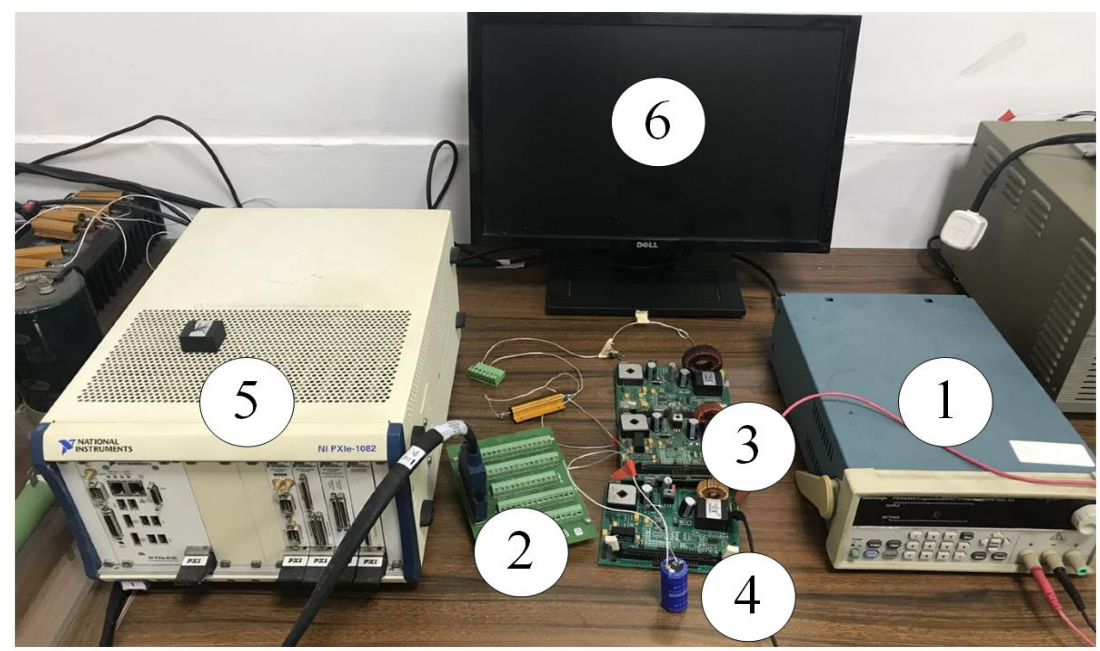

Figure 5. The hardware design: (1) power source; (2) measurement board; (3) three chargers; (4) ultracapacitor; (5) PCI extensions for Instrumentation (PXI) platform; (6) Labview. 
- $\quad$ Power source. It provides $24 \mathrm{~V}$ voltage and can power three chargers.

- Chargers. According to Figure 1, design the buck circuit for this experiment. Each charger includes a buck regulator and voltage sensor, a PWM driver TLP700A, a current sensor CSM005A, filter chip TL074ID, a voltage transition chip PDUKE-24S05, and a TMS320F2808 microprocessor. The three chargers communicate with each other via the Controller Area Network (CAN) bus.

- Ultracapacitor. We use the ultracapacitor battery as the load and use the output of the three chargers as the input. The model of the ultracapacitor is DP-2R5D607AA5, and its specific parameters are equivalent-serial-resistor (ESR) $R=5 \mathrm{~m} \Omega$ and $C=100 \mathrm{~F}$.

- PXI platform. Measure the current and voltage of the ultracapacitor through the PXI platform and use Labview to display the waveform on the computer.

The specific process of this experiment is as follows. The current sensor CSM005A is used to measure the current of the charger. The high-precision voltage divider is used to measure the output voltage of the charger. The low pass filter TL074ID filters the measured analog signal using TMS320F2808 microprocessor to process measured values. The three TMS320F2808 microprocessors communicate through the CAN module. Every controller produces a corresponding PWM signal to adjust the current and output voltage. The PXI platform is used to collect data, where the sampling frequency is $1 \mathrm{KHz}$.

\subsection{Experiment Results}

We experimentally proved the feasibility of this method. The experimental results are shown in Figures 6-9, which are introduced as follows.

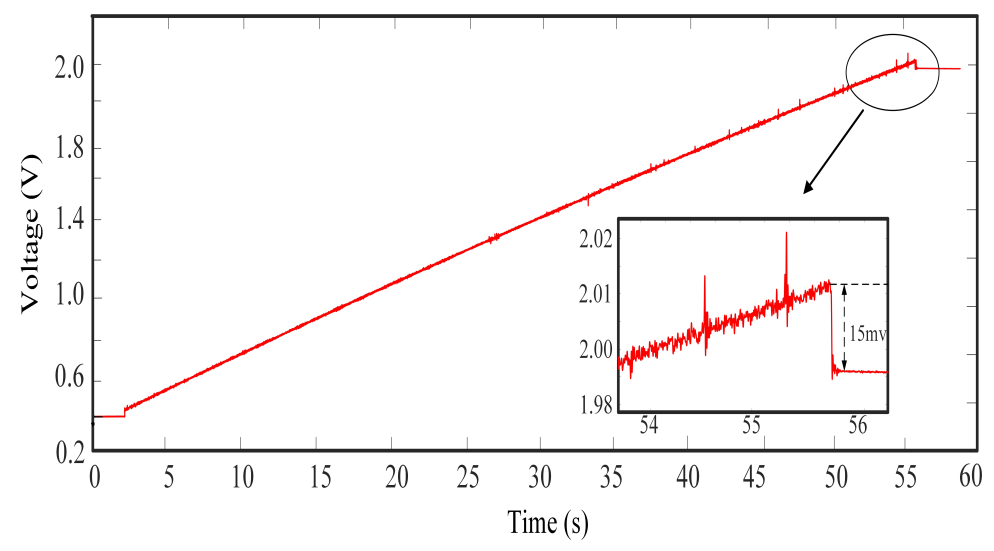

Figure 6. Ultracapacitor charging voltage.

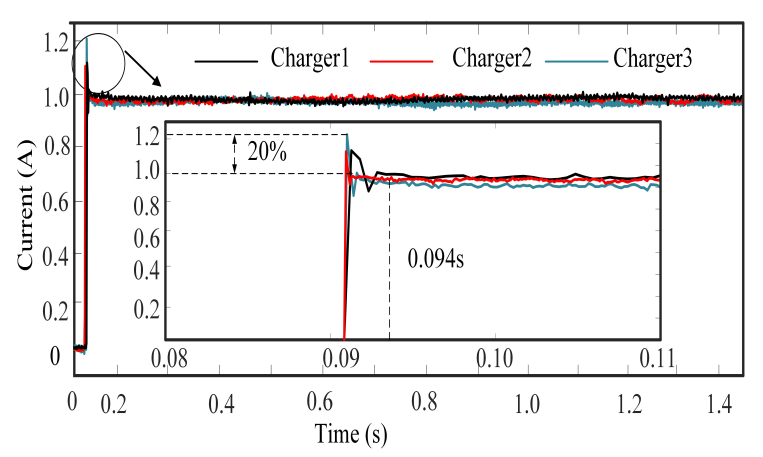

(a)

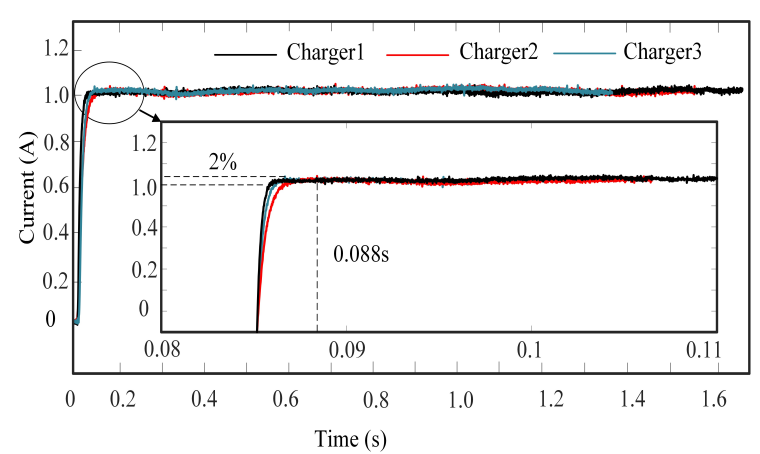

(b)

Figure 7. Experiment results of (a) the classical charging method and (b) the proposed charging method. 


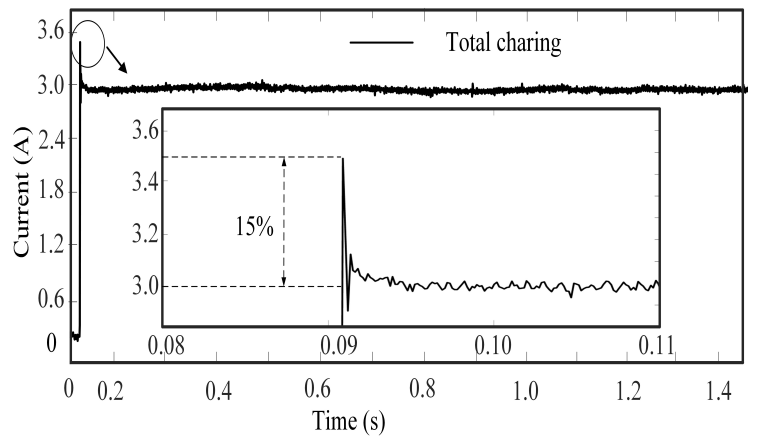

(a)

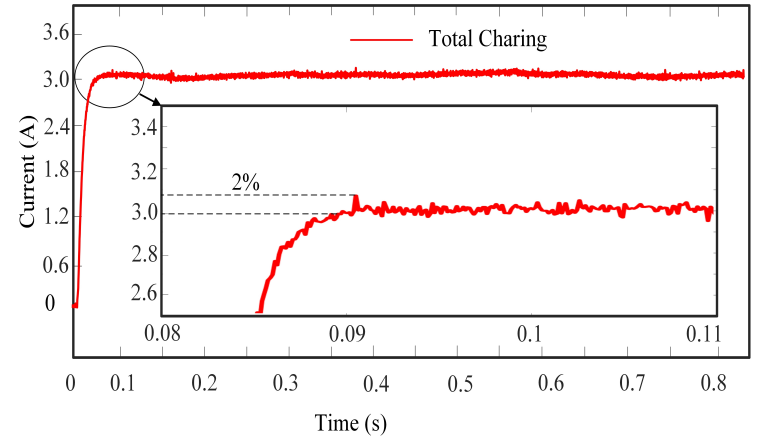

(b)

Figure 8. Experiment results of (a) the classical charging method and (b) the proposed charging method in total charging current.

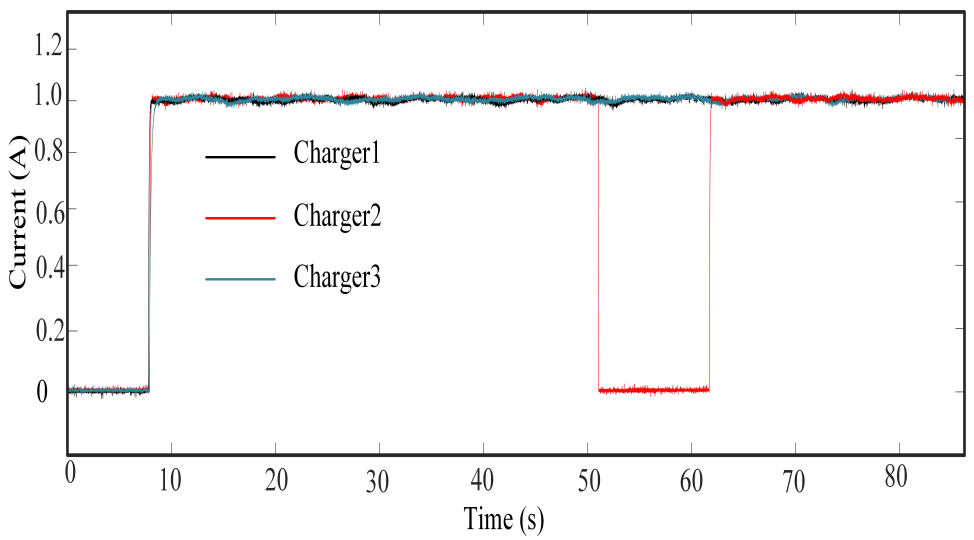

Figure 9. Charge current when charger fails.

Figure 6 depicts the voltage variation of the ultracapacitor. After being fully charged, the charging process is disconnected, and the voltage of the ultracapacitor itself will cause a voltage drop of $15 \mathrm{mV}$. The steady-state current for each shipper is $1 \mathrm{~A}$. Figure 7 shows the experiment results, which explores the effect of set point modulation on the charging process. Figure 7 a shows the experiment results of the classical charging method, where the overshoot is about $20 \%$ and the settling time is about $0.094 \mathrm{~s}$. Figure $7 \mathrm{~b}$ shows the experiment results of the proposed method, where the current overshoot is about $2 \%$ and the settling time is about $0.088 \mathrm{~s}$. Table 2 summarizes the experimental results of Figure 7, from which it can be concluded that the proposed charging method can restrain the current while reducing the settling time. Figure 9 shows the fault tolerance of the system. When one charger encounters a failure, it will not affect other chargers, and after resuming charging, there will be no overshoot. In practical applications, it can effectively extend the life of chargers and ultracapacitors.

Figure 8 shows the experiment results of the two methods in total charging current. Figure $8 \mathrm{a}$ shows the experiment results of the classical charging method, where the overshoot is about $15 \%$ and the settling time is about $0.094 \mathrm{~s}$. Figure $8 \mathrm{~b}$ shows the experiment results of the proposed charging method, where the overshoot is about $2 \%$ and the settling time is about $0.088 \mathrm{~s}$. Therefore, experiment results verify that the proposed charging method can restrain the current overshoot while reducing the settling time.

Table 2. Summaries of experiment results in Figure 7.

\begin{tabular}{ccc}
\hline Metric $\backslash$ Method & Classical Charging Method & Proposed Charging Method \\
\hline Overshooting & $20 \%$ & $2 \%$ \\
Settling Time & $94 \mathrm{~ms}$ & $88 \mathrm{~ms}$ \\
\hline
\end{tabular}




\subsection{Result Discussions}

We make the following comments about the proposed charging method.

\subsubsection{Effectiveness}

We can conclude from the two experimental results of Figure 7 that the proposed charging method can restrain the current overshoot. The overshoot of the classical charging method and the proposed charging method is $2 \%$ and $20 \%$, respectively. This is because the proposed method can adjust the reference value of the closed-loop system in time according to the actual output. When the expected output shows an excessive amount, the reference value is adjusted to a lower value to reduce the effect of overshoot.

\subsubsection{Fault Tolerance}

As shown in Figure 9, when one charger fails, there will be no interference between chargers. When the charger is restored, the charger successfully prevents overshoot from occurring, which means that the proposed charging method is able to respond quickly to changes in dynamic conditions. This verifies the fault tolerance of the proposed method.

\subsubsection{Practicality}

In the process of charging ART vehicle, the charging current is very large. So a little overshoot will cause a very large current deviation, which will cause damage to the charger and super capacitor and reduce their service life. The proposed charging method can restrain the overshoot and has positive significance for extending the life of the charger and super capacitor.

\section{Conclusions}

In this paper, we propose a predictive set point modulation charging strategy to reduce the current overshoot during the charging process. The multicharger system is modeled using the state-space averaging method. The decentralized control is applied and the closed-loop model is derived. The prediction set point modulation strategy is analyzed and then applied to improve the dynamic performance of the closed-loop charging system. A practical case study is provided to illustrate how to design the compensators. Experimental results verify that compared with the classical charging method, the current overshooting is reduced from $20 \%$ to $2 \%$ with the proposed method.

Author Contributions: Conceptualization, H.L. (Hongtao Liao) and J.P.; methodology, H.L. (Heng Li) and H.L. (Hongtao Liao); validation, Y.Z.; data curation, Y.Z. and H.L. (Hongtao Liao); writing-original draft preparation, H.L. (Heng Li) and Y.Z.; writing - review and editing, H.L. (Hongtao Liao) and J.P. All authors have read and agreed to the published version of the manuscript.

Funding: This research is supported by the National Natural Science Foundation of China (Grant No. 61803394) and the Hunan Provincial Natural Science Foundation of China (Grant No. 2019JJ50822).

Conflicts of Interest: The authors declare no conflict of interest.

\section{References}

1. He, Y.; Hildmann, M.; Herzog, F.; Andersson, G. Modeling the Merit Order Curve of the European Energy Exchange Power Market in Germany. IEEE Trans. Power Syst. 2013, 28, 3155-3164. [CrossRef]

2. Moret, F.; Pinson, P. Energy Collectives: A Community and Fairness Based Approach to Future Electricity Markets. IEEE Trans. Power Syst. 2019, 34, 3994-4004. [CrossRef]

3. Zheng, C.; Li, W.; Liang, Q. An Energy Management Strategy of Hybrid Energy Storage Systems for Electric Vehicle Applications. IEEE Trans. Sustain. Energy 2018, 9, 1880-1888. [CrossRef]

4. Dusmez, S.; Khaligh, A. A Supervisory Power-Splitting Approach for a New Ultracapacitor-Battery Vehicle Deploying Two Propulsion Machines. IEEE Trans. Ind. Inform. 2014, 10, 1960-1971. [CrossRef] 
5. Newman, P.; Hargroves, K.; Davies-Slate, S.; Conley, D.; Verschuer, M.; Mouritz, M.; Yangka, D. The trackless tram: Is it the transit and city shaping catalyst we have been waiting for? J. Transp. Technol. 2019, 9, 31-55. [CrossRef]

6. Qi, C.; Wang, K.; Yang, Q.; Li, G.; Huang, X.; Wu, J.; Crow, M.L. Decentralized DC Voltage and Power Sharing Control of the Parallel Grid Converters in Multi-Terminal DC Power Integration System. IEEE Trans. Sustain. Energy 2019, 10, 1971-1980. [CrossRef]

7. Song, J.; Lee, D.; Woo, J.; Koo, Y.; Cha, E.; Lee, S.; Park, J.; Moon, K.; Misha, S.H.; Prakash, A.; et al. Effects of RESET Current Overshoot and Resistance State on Reliability of RRAM. IEEE Electron Device Lett. 2014, 35, 636-638.

8. Shrestha, P.R.; Nminibapiel, D.M.; Campbell, J.P.; Ryan, J.T.; Veksler, D.; Baumgart, H.; Cheung, K.P. Analysis and Control of RRAM Overshoot Current. IEEE Trans. Electron Devices 2018, 65, 108-114. [CrossRef]

9. Zhang, X.; Zhang, H.; Li, H.; Jiao, Y.; Liao, H.; Peng, J.; Huang, Z. Cooperative charging of supercapacitor trams with current overshoot suppression. IEEE Trans. Ind. Appl. 2020, 56, 4155-4165. [CrossRef]

10. Ghaffarzadeh, H.; Stone, C.; Mehrizi-Sani, A. Predictive set point modulation to mitigate transients in lightly damped balanced and unbalanced systems. In Proceedings of the 2017 IEEE Power Energy Society General Meeting, Chicago, IL, USA, 16-20 July 2017; p. 1.

11. Mehrizi-Sani, A.; Iravani, R. Online Set Point Modulation to Enhance Microgrid Dynamic Response: Theoretical Foundation. IEEE Trans. Power Syst. 2012, 27, 2167-2174. [CrossRef]

12. Zhou, Y.; Huang, Z.; Liao, H.; Li, H.; Jiao, Y.; Peng, J. A Predictive Set-Point Modulation Energy Management Strategy for Hybrid Energy Storage Systems. IEEE Trans. Ind. Appl. 2019, 55, 6266-6277. [CrossRef]

13. Mehrizi-Sani, A.; Iravani, R. Online Set Point Adjustment for Trajectory Shaping in Microgrid Applications. IEEE Trans. Power Syst. 2012, 27, 216-223. [CrossRef]

14. Li, H.; Zhang, X.; Peng, J.; He, J.; Huang, Z.; Wang, J. Cooperative CC-CV Charging of Supercapacitors Using Multi-Charger Systems. IEEE Trans. Ind. Electron. 2020, 67, 10497-10508. [CrossRef]

15. Zhang, W.; Tian, W.; Sun, Z.; Shi, L. New charging system study of energy storage trams. Power Electron. 2018, 52, 58-60.

16. Wang, J.M.; Tzeng, L.; Hsu, M.T.; Jian, H.R. A Simple Control Scheme to Avoid the Sensing Noise for the DC-DC Buck Converter With Synchronous Rectifier. IEEE Trans. Ind. Electron. 2018, 65, 5086-5091. [CrossRef]

17. Erickson, R.W.; Maksimovic, D. Fundamentals of Power Electronics; Springer Science \& Business Media: New York, NY, USA, 2007.

18. Mousavi, S.M.H.; Makki, S.V.A.; Siahkamari, H.; Alirezaee, S.; Ahmadi, M. Performance Improvement of Microstrip LPF Based on Transfer Function Analysis. IEEE Microw. Wirel. Components Lett. 2016, 26, 322-324. [CrossRef]

19. Gómez, P.; Vergara, L.; Nuricumbo-Guillén, R.; Espino-Cortés, F.P. Two-Dimensional Definition of the Numerical Laplace Transform for Fast Computation of Transient Profiles Along Power Transmission Lines. IEEE Trans. Power Deliv. 2016, 31, 412-414. [CrossRef]

20. Tsui, C.-C. High-performance state feedback, robust, and output feedback stabilizing control-a systematic design algorithm. IEEE Trans. Autom. Control 1999, 44, 560-563. [CrossRef]

21. Ang, K.H.; Chong, G.; Li, Y. PID control system analysis, design, and technology. IEEE Trans. Control Syst. Technol. 2005, 13, 559-576.

22. Harnefors, L. Modeling of Three-Phase Dynamic Systems Using Complex Transfer Functions and Transfer Matrices. IEEE Trans. Ind. Electron. 2007, 54, 2239-2248. [CrossRef]

23. Ayasun, S.; Nwankpa, C.O.; Kwatny, H.G. Voltage Stability Toolbox for Power System Education and Research. IEEE Trans. Educ. 2006, 49, 432-442. [CrossRef]

24. Phillips, C.L.; Parr, J.M. An approximation for the damping ratio zeta in a second-order system. IEEE Trans. Educ. 1991, 34, 145-146. [CrossRef]

(C) 2020 by the authors. Licensee MDPI, Basel, Switzerland. This article is an open access article distributed under the terms and conditions of the Creative Commons Attribution (CC BY) license (http:/ / creativecommons.org/licenses/by/4.0/). 